\title{
Marital status, depressive episodes, and short- term prognosis of patients with acute coronary syndrome: Greek study of acute coronary syndrome (GREECS)
}

\author{
Demosthenes B \\ Panagiotakos ${ }^{1,3}$ \\ Christos Pitsavos ${ }^{2,3}$ \\ Yannis Kogias ${ }^{3}$ \\ Yannis Mantas ${ }^{3}$ \\ Spyros Zombolos ${ }^{3}$ \\ Antonis Antonoulas ${ }^{3}$ \\ George Giannopoulos ${ }^{2}$ \\ Christina Chrysohoou ${ }^{2}$ \\ Christodoulos Stefanadis' \\ 'Office of Biostatistics - Epidemiology, \\ Department of Nutrition - Dietetics, \\ Harokopio University, Athens, \\ Greece; ${ }^{2}$ First Cardiology Clinic, \\ School of Medicine, University of \\ Athens, Greece; ${ }^{3}$ The GREECS Study \\ Investigators, Greece
}

\begin{abstract}
The association between marital status and short-term prognosis of patients hospitalized for acute coronary syndrome (ACS) was evaluated. From October 2003 to September 2004, a sample of 6 hospitals located in Greek regions was selected, and almost all survivors after an ACS were enrolled into the study (2172 patients were included in the study; $76 \%$ were men). The in-hospital mortality rate was $3.2 \%$ in male patients and $5.7 \%$ in female patients $(\mathrm{p}=0.009)$. Never-married patients had 2.8-times higher risk of dying during hospitalization compared with married, after adjusting for various confounders $(\mathrm{p}<0.01$, attributable risk $=$ 64\%). Furthermore, never-married had 2.7-times higher risk of dying during the first 30-days following hospitalization compared with married $(\mathrm{p}<0.01$, attributable risk $=62 \%)$. Moderate depressive symptoms 3.26-fold (95\% CI 1.40-7.11) the risk of recurrent events, while severe depressive symptoms were associated with 8.2-fold (95\% CI 3.98-17.1) higher risk of events. No interaction was observed between marital status and depression on 30-day prognosis of ACS patients $(\mathrm{p}>0.5)$. People who were not-married and depressed at the time of an acute cardiac episode were at higher risk of fatal events than people who were married, irrespective of depression status and other characteristics.
\end{abstract}

Keywords: marital status, acute coronary syndromes, risk

\section{Introduction}

It has been reported in many epidemiologic studies that social factors, like marital status, may predict cardiovascular mortality and morbidity in both genders (Weiss et al 1973; Slater et al 1988; Mendes et al 1992; Gallo et al 2003; Steptoe et al 2004; Nilsson et al 2005; Birkenaes et al 2006). For example, in the Malmo Preventive project (Nilsson et al 2005) where the investigators studied information from about 53,000 males and females from Sweden, it was found that increased mortality risk was documented for subjects who were divorced or unmarried, even after adjusted for social class. In another recent study, Gallo and colleagues (2003), reported that women in satisfying marriages had the least atherosclerosis in the carotid arteries and aorta, especially relative to those in low-satisfying marriages. Furthermore, women in satisfying marriages also tended to show less rapid progression of carotid atherosclerosis relative to women in low-satisfying marriages, while women who did not have a partner had intermediate levels of atherosclerosis. Few years before, in the Minnesota Health Survey, Venters and colleagues (1986) studied 7850 men and women and reported that divorced persons had the highest rates of hospitalization for heart disease or stroke, while married and widowed persons had the lower and intermediate rates, respectively, of such hospitalization. Moreover, never-married reported 
hospitalization lowers than those of married persons. Further analysis revealed that divorced persons had higher levels of psychological disorders, smoking, drinking, and physical activity than married; however, married men, had higher levels of total cholesterol and lower levels of serum high-density lipoprotein cholesterol. The investigators of the Minnesota Health Survey concluded that marital status is important in identifying, understanding, and altering behavior known to increase risk for cardiovascular disease (CVD) (Venters et al 1986). The US Department of Health and Human Services indicates that married people are healthier than other adults (Focus on the Family 2006). This was supported by the fact that married people had fewer limitations in daily activities and in physical or social functioning; they were less likely to suffer from severe psychological stress, to have a physically inactive lifestyle, to smoke, and to engage in heavy alcohol drinking.

Although the relationship between marital status, the levels of cardiovascular risk factors, and the profile of cardiac patients has been investigated in several studies, the association between being married, divorced, widowed or nevermarried with the short-term prognosis after a hard cardiac event has rarely been ascertained, especially in the light of psychological disorders. In the context of the GREECS study we assessed the relationship between marital and depression status, in-hospital mortality and 30-day outcome (ie, death or rehospitalization due to cardiovascular events) of patients who had had an acute cardiac event.

\section{Methods}

\section{Study sample}

From October 2003 to September 2004 almost all $(n=2172)$ consecutive patients with discharge diagnosis of ACS (acute myocardial infarction $[\mathrm{MI}]$ or unstable angina [UA]) that were hospitalized in the cardiology clinics or the emergency units of six major General Hospitals in Greece (ie, Hippokration hospital in Athens and the general prefecture hospitals in Lamia, Karditsa, Halkida, Kalamata, and Zakynthos island) were enrolled into the study (the participation rate varied from $80 \%$ to $95 \%$ ). The hospitals were selected in order to represent populations with various socioeconomic, cultural, and regional characteristics. Of the enrolled patients, $1649(76 \%)$ were men and 523 (24\%) were women. With the exception of Athens - where there are several other hospitals - all the other hospitals cover the whole population of the aforementioned regions, including urban and rural areas.

At entry, as well as during hospitalization (at 6 to $9 \mathrm{~h}$, and again at 12 to $24 \mathrm{~h}$ if earlier samples were negative and the clinical index of suspicion was high) measurements of troponin I, creatinin phosphokinase (CK), and the MB fraction of total creatinine phosphokinase (CK-MB) were obtained from all patients to detect evidence of myocardial cell death (ESC/ACC 2000). Moreover a 12-lead electrocardiogram (ECG) was performed and clinical symptoms were evaluated in all patients, by a cardiologist of the study. Acute MI was defined by typical rise and gradual fall (troponin I) or more rapid rise and fall (CK-MB) of biochemical markers of myocardial necrosis (troponin I $>0.4 \mathrm{ng} / \mathrm{ml}$ and the MB fraction of CK-MB $>8.8 \mathrm{ng} / \mathrm{ml}$ ) with at least one of the following: (a) ischemic symptoms, (b) development of pathologic Q waves on the ECG, (c) ECG changes indicative of ischemia (ST segment elevation or depression) or (d) coronary artery intervention (eg, coronary angioplasty) (Alpert et al 2000). UA was defined by the occurrence of one or more angina episodes, at rest, within the preceding 48-hours, corresponding to class III of the Braunwald classification.

The study was approved by the Medical Research Ethics Committee of our Institution and was carried out in accordance with the Declaration of Helsinki (1989) of the World Medical Association.

\section{Investigated measurements}

After the third day of hospitalization and when the clinical condition was stable information from 2157 patients ( 15 died within 24 hours of hospitalization) regarding nutritional and other lifestyle habits, as well as various sociodemographic characteristics, was retrieved. Medical information was recorded through patient's hospital records. In $4 \%$ of cases $(n=87)$ where the patients were unable to provide accurate information, this information was obtained from people who accompanied them, if they were aware of patients' medical history, as well as their lifestyle and behavioral habits. Thus, by the exception of 15 patients who died during the first 24 hours of their admission, information was obtained from all other participants.

\section{Sociodemographic characteristics}

Marital status was recorded in 2090 participants and classified in the following four categories: (a) married at the time of the event $(\mathrm{n}=1711)$, (b) never married $(\mathrm{n}=102)$, (c) divorced $(\mathrm{n}=57)$, and $(\mathrm{d})$ widowed $(\mathrm{n}=220)$. The last two categories were combined in all analyses because of the small sample size of the group.

Other sociodemographic characteristics included: age, sex, number of children, years of school, type of occupation, and mean annual income of the family (through self reports) 
during the last three years. Regarding people in the family who were not working, the average family income was used, while for unemployed individuals the basic monthly allowance they take from the Social Service Office was recorded. Participants were then classified as low $(<12,000$ euros $)$, moderate (12,000 to 18,000 euros), and high $(>18,000$ euros) financial status group according to their reports. Height and weight was measured to the nearest $0.5 \mathrm{~cm}$ and 100 gr, respectively.

\section{Anthropometric indices and medical characteristics}

Body mass index (BMI) was then calculated as weight (in kilograms) divided by height (in meters) squared. Overweight was defined as BMI between 25 and $29.9 \mathrm{~kg} / \mathrm{m}^{2}$, while obesity as BMI greater than $29.9 \mathrm{~kg} / \mathrm{m}^{2}$. Furthermore, a detailed medical history was recorded, including previous hospitalization for CVD (ie, coronary heart disease, stroke, or other CVD), presence and management of hypertension, hypercholesterolemia, and diabetes mellitus. Moreover, it was recorded patients' medical family history.

\section{Lifestyle and dietary characteristics}

To evaluate physical activity status of the patients during the past year, a modified version of a self-reported questionnaire provided by the American College of Sports Medicine was applied (Pate et al 1995). Based on this questionnaire the frequency (times per week), duration (in minutes per time) and intensity of sports or occupation related physical activity was recorded. Participants who did not report any physical activities were defined as physically inactive (ie, sedentary). Current smokers were defined as those who smoked at least one cigarette per day or have stopped cigarette smoking during the past 12 months. Former smokers were defined as those who had stopped smoking more than one year ago. The rest of them were defined as never smokers or rare smokers. Usual dietary intake over the year preceding enrolment was assessed by a semi-quantitative food frequency questionnaire that included various foods and beverages commonly consumed in Greece. Then, the frequency of consumption was quantified approximately in terms of the number of times a month this food was consumed. Thus, daily consumption multiplied by 30 and weekly consumption multiplied by 4 and a value of 0 was assigned to food items rarely or never consumed. Alcohol consumption was measured in wineglasses (100 $\mathrm{ml}$ ) and quantified by ethanol intake (grams per drink). One wineglass was equal to 12 gr ethanol concentration. Based on the Mediterranean diet pattern and the reported monthly frequency consumption of these food groups, a special diet score was calculated for each participant that assessed adherence to the Mediterranean diet (range 0-55). In particular, 10 food groups were evaluated: non-refined cereals and products, fruits and nuts, vegetables, olive oil, nonfat or low fat dairy, fish, poultry, potatoes, pulses, red meat, or meat products and eggs, as well as alcohol consumption. The diet score was calculated as follows: for the consumption of items presumed to be close to this pattern (ie, nonrefined cereals and products, fruits and nuts, vegetables, olive oil, nonfat or low fat dairy, fish, potatoes, and pulses) scores 0 to 5 were assigned when a participant reported no consumption to daily consumption, while for the consumption of foods presumed to be away from this diet (like meat or meat products, eggs, and poultry) reverse scores were assigned (ie, score 0 when a participant reported almost daily consumption to score 5 for rare or no consumption). Especially for alcohol score 5 was assigned for consumption of 1 to 3 wineglasses per day, score 0 for consumption of more than 7 wineglasses per day and scores 1 to 4 for consumption of 0,4 to 5, 6 and 7 wineglasses per day. Higher values of this diet score indicate greater adherence to the Mediterranean diet.

\section{Psychological evaluation}

Clinical symptoms of depression during the past month was determined by a special, confidential and weighted questionnaire, which was based on a self-reported depression scale (range 0-60) developed by Radloff, known as the Center of Epidemiological Studies-Depression scale (CES-D) (Radloff 1977). The containment of the investigated emotions, are listed below: "How often during the past month you..." were bothering by things that usually do not bother you; had poor appetite; feeling that you could not shake off the blues even with help from your family; feeling of being just as good as other people; trouble keeping your mind on what you were doing; feeling that everything you did was an effort; feeling hopeful about your future; thought that your life had been a failure; feeling fearful; having restless sleep; being happy; talking less than usual; feeling lonely; considering people being unfriendly to you; enjoying your life; having crying spells; feeling sad; feeling that people disliked you; could not get "going".

Further details about the methodology used for the study have been presented elsewhere (Pitsavos et al 2005).

\section{Statistical analysis}

Continuous variables are presented as mean values \pm standard deviation, while categorical variables are presented as 
absolute and relative ( $\%$ ) frequencies. Associations between normally distributed continuous variables and marital status group of patients were evaluated through the analysis of variance (ANOVA), after controlling for equality of variances (homoscedacity). However, the associations between skewed variables and group of patients were evaluated through the Kruskal-Wallis test. Due to multiple comparisons the Bonferroni rule was applied to correct for the inflation of Type-I error. Associations between categorical variables were tested by the use of the chi-squared test, without the correction of continuity. Correlations between continuous variables were evaluated by the use of Pearson's correlation coefficient for the normally distributed and by the use of Spearman's rho coefficient for the ordinal or skewed variables. Normality was evaluated through the Shapiro-Wilk test, while homoscedacity and serial dependency of the response variables was graphically assessed (standardized residuals versus fitted values). The association between the investigated marital status and the in-hospital mortality or 30-day outcome (ie, death or rehospitalization due to CVD) was assessed by the use of multiple logistic regression analysis, after controlling for sociodemographic, clinical, and biochemical characteristics of the patients. Appropriate tests for goodness-of-fit (ie, deviance and Pearson's residuals) were applied in all models. The attributable fraction (AF) was estimated by the equation: $\mathrm{AF}=($ odds ratio -1$)$ /odds ratio. A value of false positive (type I) error less than $5 \%$ was considered as statistically significant. All statistical calculations will be performed on the SPSS version 12.0 software (SPSS Inc, Chicago, Il, USA).

\section{Results}

From October 2003 to September 2004, 2172 patients with discharge diagnosis of ACS were enrolled into the study (1649 men, $65 \pm 13$ years old and 523 women, $72 \pm 11$ years old, $\mathrm{p}<0.001)$. According to the discharge diagnosis, $764(35 \%)$ patients were diagnosed as having unstable angina, 699 (32\%) patients as having non-Q-wave MI and 709 (33\%) patients as having Q-wave MI. Of the patients 1711 were married at the time of event, while the rest of them were widowed, divorced, or never-married. Table 1 illustrates various sociodemographic, lifestyle and behavioral characteristics of the patients by marital status group. It was observed that divorced or widowed were more likely to be females compared with married or never-married. Moreover, never-married were younger, consumed lower quantities of alcoholic beverages, were physically inactive, smokers, but also had higher education compared with married or divorced/widowed (Table 1). No differences were observed between marital status groups regarding dietary habits and financial status.

The mean depression (CES-D) score was $13.5 \pm 12$ in male patients and $13.6 \pm 12$ in females $(p=0.89)$. Compared with patients with UA those who had non-Q-wave MI or Q-wave MI had higher CES-D score (12.3 \pm 11.6 vs. $15.3 \pm$ 12.8 vs. $13.2 \pm 11.9, \mathrm{p}=0.001)$; moreover, patients with non-Q-wave MI had higher CES-D score compared with those with Q-wave MI ( $\mathrm{p}=0.004)$.

The in-hospital mortality rate was $3.2 \%$ in male and $5.7 \%$ in female patients (ie, overall 82 deaths, $\mathrm{P}$ for gender differences $=0.009$ ). At this point it should be mentioned that 15 of 82 patients who died during hospitalization did not fulfill the questionnaire, thus they excluded from the analyses. During the first 30-days following discharge precise information about vital status or rehospitalization due to CVD retrieved from 1680 patients (77\% participation rate). The number of events during this short follow-up period was: 119 in males $(9.2 \%)$ and 38 in females $(9.7 \%)$, while $41(26 \%)$ of the events were fatal (25 in males, $\mathrm{p}$ for gender differences $=0.017$ ).

Table 2 illustrates various clinical characteristics of the patients by marital status. Never married were less frequently to have hypertension, diabetes, and previous CVD compared with married or divorced/widowed. However, these differences were explained when the analysis was adjusted for age and sex. Additionally, married or never married were more likely to have MI, while widowed or divorced had more frequently UA. No differences were observed between marital status groups and adherence to treatment of various co-morbidities, as well as presence of depression (Table 2).

Regarding the prognosis of ACS patients, married had lower fatal event rates during hospitalization, as well as within 30-days after discharge, compared with never-married or divorced/widowed. In particular, the age-sex adjusted inhospital mortality ratio between never-married and married was 2.3 ( $p=0.03)$, while the 30-day death ratio between the aforementioned groups was $2.0(p=0.01)$. Moreover, divorced or widowed had more fatal events, were more likely to have hypertension, and diabetes compared with married (Table 2).

Although the differences between marital status groups and in-hospital or 30-day outcome of the patients were adjusted for age and sex, several other potential confounders may exist (eg, differences in clinical status at presentation). Table 3 presents the results from multiple logistic regression 
Table I Sociodemographic, lifestyle and behavioral characteristics by marital status

\begin{tabular}{|c|c|c|c|c|}
\hline & Never-married $(\mathrm{n}=102)$ & Married $(n=|7| I)$ & $\begin{array}{l}\text { Divorced/widowed } \\
(n=277)\end{array}$ & $\mathbf{p}$ \\
\hline Male sex (\%) & 89 & 81 & $4 I^{* *}$ & 0.001 \\
\hline Age (years) & $56 \pm 14$ & $65 \pm 12^{* *}$ & $74 \pm 12^{* *}$ & 0.001 \\
\hline Number of children & $0.3 \pm 0.6$ & $2.2 \pm 1 . I^{* *}$ & $2.4 \pm 1.5^{* *}$ & 0.001 \\
\hline \multicolumn{5}{|l|}{ Financial status } \\
\hline Low or moderate & $68 \%$ & $67 \%$ & $72 \%$ & 0.32 \\
\hline High or very high & $32 \%$ & $33 \%$ & $28 \%$ & \\
\hline Diet score $(0-55)$ & $27.7 \pm 5.1$ & $28.8 \pm 5.7$ & $28.2 \pm 5.6$ & 0.42 \\
\hline Alcohol drinking ( $>4$ wineglasses/d, \%) & 19 & $26 * *$ & $24^{*}$ & 0.001 \\
\hline Physical inactivity (\%) & 43 & 40 & $32^{*}$ & 0.03 \\
\hline Current smoking (\%) & 46 & $32 *$ & $24 * *$ & $<0.001$ \\
\hline Years of school & $9.2 \pm 4.7$ & $7.8 \pm 4.2^{*}$ & $6.6 \pm 4.6 * *$ & $<0.001$ \\
\hline
\end{tabular}

Notes: ${ }^{*} \mathrm{p}<0.05 ;{ }^{* *} \mathrm{p}<0.01$ between married, divorced/widowed vs. never-married group, after correcting for multiple comparisons through the Bonferroni correction.

analysis that evaluated the association between marital status and in-hospital mortality after adjusting for age, sex, discharge diagnosis, smoking and eating habits, clinical characteristics (eg, hypertension, hypercholesteremia, diabetes, previous CHD, and family history of cardiac disease), physical activity, and education status. The analysis showed that never-married had 2.8-times higher odds of dying after hospitalization compared with married, after adjusting for the aforementioned confounders. The AF was calculated equal to $64 \%$. Being divorced or widowed tended to increase the likelihood of fatal events within hospital, however this association did not reach significance.

Table 2 Prevalence of clinical characteristics and short term prognosis by marital status

\begin{tabular}{|c|c|c|c|c|}
\hline & Never-married $(n=102)$ & Married $(n=|7| I)$ & Divorced/widowed $(n=277)$ & $\mathbf{p}$ \\
\hline Obesity (\%) & 18 & 23 & 24 & 0.41 \\
\hline Hypertension (\%) & $44^{\dagger}$ & 50 & $67^{t \dagger}$ & 0.001 \\
\hline Treated (\% of hypertensive) & 78 & 78 & 79 & 0.15 \\
\hline Hypercholesterolemia (\%) & 47 & 47 & 44 & 0.68 \\
\hline Treated (\% of hypercholesterolemic) & 38 & 39 & 50 & 0.12 \\
\hline Diabetes mellitus (\%) & $26^{\dagger}$ & 31 & $40^{\dagger \dagger}$ & 0.005 \\
\hline Treated (\% of diabetic) & 67 & 70 & 75 & 0.67 \\
\hline Prior CVD (\%) & 31 & 40 & 52 & 0.001 \\
\hline Family history of CHD (\%) & 42 & 37 & 34 & 0.39 \\
\hline CES-Depression score $(0-60)$ & $16 \pm 9$ & $13 \pm 9$ & $1 \mathrm{II} 8$ & 0.11 \\
\hline Discharge diagnosis & & & & 0.02 \\
\hline Q-wave MI (\%) & 37 & 33 & $25^{\dagger}$ & \\
\hline Non-Q-wave MI (\%) & 30 & 32 & 32 & \\
\hline Unstable angina (\%) & 33 & 35 & $43^{\dagger}$ & \\
\hline \multicolumn{5}{|l|}{ Short term prognosis } \\
\hline In-hospital deaths (\%) & $7^{\dagger}$ & $3^{*}$ & $5 * \dagger$ & 0.03 \\
\hline 30-day nonfatal events (\%) & 4 & 7 & 9 & 0.28 \\
\hline 30-day fatal events (\%) & $4^{\dagger}$ & $2^{*}$ & $5^{\dagger}$ & 0.01 \\
\hline $\begin{array}{l}\text { Adherence to treatment after } \\
\text { discharge (\%) }\end{array}$ & 78 & 86 & 82 & 0.13 \\
\hline
\end{tabular}

Notes: ${ }^{*} \mathrm{p}<0.05 ;{ }^{*} \mathrm{p}<0.01$ between married, divorced/widowed vs. never-married group, after correcting for multiple comparisons through the Bonferroni correction; ${ }^{\dagger} \mathrm{p}<0.05 ;{ }^{\dagger \dagger} \mathrm{p}<0.0$ l between never-married, divorced/widowed vs. married group, after correcting for multiple comparisons through the Bonferroni correction. 
Table 3 Results from the multiple logistic regression analysis that evaluated the association of marital status on in-hospital mortality of ACS patients

\begin{tabular}{lll}
\hline & Odds ratio & $\begin{array}{l}95 \% \text { confidence } \\
\text { interval }\end{array}$ \\
\hline Married (reference category) & 1.00 & - \\
$\quad$ Never-married & 2.80 & $2.30-3.40$ \\
$\quad$ Widowed/divorced & 1.07 & $0.45-2.5 \mathrm{I}$ \\
Age (per year) & 1.08 & $1.04-1.1 \mathrm{I}$ \\
Male vs female sex & 0.66 & $0.3 \mathrm{I}-1.39$ \\
Discharge diagnosis (MI vs UA) & 3.75 & $2.26-6.19$ \\
Current smoking (yes/no) & 1.05 & $0.43-2.6 \mathrm{I}$ \\
Hypertension (yes/no) & 0.79 & $0.41-1.55$ \\
Hypercholesterolemia (yes/no) & 1.87 & $0.96-3.64$ \\
Diabetes mellitus (yes/no) & 2.69 & $1.40-5.17$ \\
Family history of CHD (yes/no) & 1.37 & $0.64-2.35$ \\
Previous CVD (yes/no) & 0.47 & $0.22-0.97$ \\
Obesity (yes/no) & 0.67 & $0.28-1.58$ \\
Physical activity (yes/no) & 0.54 & $0.25-1.58$ \\
Education (per I-year of school) & 1.02 & $0.92-1.14$ \\
\hline
\end{tabular}

Abbreviations: ACS, acute coronary syndrome; CVD, cardiovascular disease; $\mathrm{CHD}$, coronary heart disease; $\mathrm{MI}$, myocardial infarction; UsA, unstable angina.

No association was observed between depression status and in-hospital mortality in both genders.

Furthermore, as we can see in Table 4, never-married patients had 2.7-times higher odds of dying during the first 30-days following hospitalization, compared with married, after adjusting for various potential confounders (Table 4). The AF was $62 \%$. Additionally, severe depressive symptoms were associated with 10.1-times higher odds for death (95\% CI 2.3-43.4), after adjusting for the aforementioned factors, while moderate or severe depression was associated with 2.1- (95\% CI 1.0-4.3) and 5.4-times (95\% CI 2.7-10.5) higher odds for rehospitalization, respectively. The AF of severe depressive symptoms was $45 \%$ for death during the first 30-days following discharge, and 39\% for rehospitalization. However, no significant association was observed between depression and marital status on the investigated outcomes. Moreover, no association was observed between marital status and presence of nonfatal events during the 30-day follow-up period. Additionally, the gender-to-marital status interaction was not significant regarding the investigated outcomes (ie, in-hospital mortality or 30-day event rates). This means that being never-married has the same effect on the prognosis of ACS patients in both genders.

\section{Discussion}

The findings of the present work suggest that marital status influences the prognosis of people who had had an acute cardiac event, irrespective of depression status and other characteristics. Particularly, studying 2172 patients who were hospitalized for acute MI or UA it was revealed that compared to married, never-married experience about 2 -fold higher odds of events during hospitalization or within 30-days following discharge, irrespective of age, sex, clinical status, and lifestyle habits. This is the first Mediterranean study that reports an association between marital status and prognosis of patients who had an acute cardiac event. It is known that in Mediterranean region, where the majority of people are catholic or orthodox, marriage is strongly related to religion. Being married has been associated with financial stability, opportunities for social development, better dietary habits, and well-being. However, the presented findings were independent from psychological factors, like depression status, financial status, and dietary habits.

Many researchers have consistently demonstrated that the married people enjoy a health advantage over the unmarried (Hu and Goldman 1990; Hemstrom 1996). For example, married people report better self-assessed health, have lower rates of long-term illness, and live longer than their unmarried counterparts (Idler and Angel 1990). The association between marital status, health and mortality has been demonstrated for both genders, for Caucasian and African Americans, as well as other ethnic groups. Moreover, the health benefits of marriage are independent from various sociodemographic characteristics known to affect both marriage and health (eg, economic wellbeing, age, education level, and financial status). In the present work, studying approximately 2200 patients who had had an ACS it was observed that compared with married, divorced, or widowed, never-married patients were less frequently to have various risk factors associated with CVD such as hypertension or diabetes. However, it should be underlined that these differences were mainly attributed to age and sex differences between marital status groups. Moreover, no differences were found regarding adherence to the medication of various comorbidities such as hypertension, hypercholesterolemia, diabetes, or previous CHD. Psychological status, as measured by the depression scale, seems to be worsened among nevermarried participants, but this finding was not significant (Table 2). Regarding the lifestyle habits that are associated with the prognosis of CVD, it was observed that never-married were more likely to be sedentary and smokers. All the observed associations favor the hypothesis that never-married patients have worse health status and more health problems than 
Table 4 Results from the multiple logistic regression analysis that evaluated the association of marital status on 30-day fatal and nonfatal events of ACS patients

\begin{tabular}{|c|c|c|}
\hline & Odds ratio & $\begin{array}{l}95 \% \text { confidence } \\
\text { interval }\end{array}$ \\
\hline \multicolumn{3}{|l|}{ Fatal events } \\
\hline \multicolumn{3}{|l|}{ Marital status } \\
\hline Married (reference category) & 1.00 & - \\
\hline Never-married & 2.70 & $1.82-3.99$ \\
\hline Widowed/divorced & 1.21 & $0.42-3.53$ \\
\hline Age (per year) & 1.08 & $1.03-1.13$ \\
\hline Male vs female sex & 0.47 & $0.17-1.28$ \\
\hline Discharge diagnosis (MI vs UA) & 1.98 & $1.13-3.49$ \\
\hline Current smoking (yes/no) & 2.35 & $0.82-6.77$ \\
\hline Hypertension (yes/no) & 1.36 & $0.54-3.45$ \\
\hline Hypercholesterolemia (yes/no) & 1.15 & $0.48-2.73$ \\
\hline Diabetes mellitus (yes/no) & 1.04 & $0.48-2.73$ \\
\hline Family history of CHD (yes/no) & 1.37 & $0.64-2.35$ \\
\hline Previous CVD (yes/no) & 0.47 & $0.22-0.97$ \\
\hline Obesity (yes/no ) & 1.46 & $0.57-3.76$ \\
\hline Physical activity (yes/no) & 0.46 & $0.16-1.32$ \\
\hline Education (per I-year of school) & 1.02 & $0.92-1.14$ \\
\hline \multicolumn{3}{|l|}{ Nonfatal events } \\
\hline \multicolumn{3}{|l|}{ Marital status } \\
\hline Married (reference category) & 1.00 & \\
\hline Never married & 1.07 & $0.4 I-2.82$ \\
\hline Widowed/divorced & 1.21 & $0.42-3.53$ \\
\hline Age (per year) & 1.03 & $1.01-1.05$ \\
\hline Male vs female sex & 1.15 & $0.69-1.94$ \\
\hline Discharge diagnosis (MI vs UA) & 1.23 & $1.00-1.66$ \\
\hline Current smoking (yes/no) & 1.46 & $0.90-2.38$ \\
\hline Hypertension (yes/no) & 0.68 & $0.45-1.05$ \\
\hline Hypercholesterolemia (yes/no) & 1.68 & $1.13-2.52$ \\
\hline Diabetes mellitus (yes/no) & 1.64 & $1.09-2.49$ \\
\hline Family history of CHD (yes/no) & 1.47 & $0.69-2.50$ \\
\hline Previous CVD (yes/no) & 0.45 & $0.20-0.91$ \\
\hline Obesity (yes/no) & 0.73 & $0.44-1.22$ \\
\hline Physical activity (yes/no) & 0.63 & $0.4 I-0.97$ \\
\hline Education (per I-year of school) & 1.04 & $0.90-1.19$ \\
\hline
\end{tabular}

Abbreviations: ACS, acute coronary syndrome; CVD, cardiovascular disease; $\mathrm{CHD}$, coronary heart disease; $\mathrm{MI}$, myocardial infarction; UA, unstable angina.

married. The previous relationships may also support the finding that never-married were more likely to experience a MI, while widowed or divorced had more frequently UA. All these may support the finding that never-married had 2-times higher likelihood of having a fatal event during hospitalization or 30-days after discharge compared with married. However, it was also observed that never-married had higher education, consumed lower quantities of alcoholic beverages, and reported similar dietary habits compared with married or divorced/ widowed (Table 1). Additionally, no differences were observed regarding the financial status between marital status groups. Moreover, no differences were found between marital status groups and adherence to medication (Table 2). These results seems to support the null hypothesis (ie, marital status does not affect cardiovascular risk), instead of the alternative. All these diverse relationships make the investigated hypothesis more complicated.

It is widely adopted that, although, marital status has been linked to physical health, little agreement exists on the specific processes that account for the better health of the married compared to the never married. Theoretical explana tions for the associations between marital status and health usually take one of two forms. The one is the marital resource model which suggests that the beneficial effects of marriage on health are largely due to the greater economic resources and social support that the married enjoy, as well as to the social control that spouses exercise over the health of one another (Mirowsky and Ross 1986; Umberson 1992; Murphy et al 1997). In the present work, the effect of financial status on the relationship between marital status and short-term prognosis of ACS patients was highly insignificant. In contrast to the previous theory, the stress model suggests that the strains of marital dissolution undermine health and well-being more than the resources of marriage protect it (Booth and Amato 1991; Williams 1992). Thus, the better health status of the married compared with the divorced or widowed reflects the negative health consequences of marital loss than of the benefits of being married. Recently, Eng and colleagues (1992) studied approximately 39,000 elderly men from the US Health Professionals Study and observed that marital termination affects health and dietary behaviors. In particular, compared with men who were married for at least four years, men who became widowed increased their alcohol consumption, decreased their BMI, decreased vegetable intake, while remarriage was linked to greater consumption, and increased levels of psychological stress. In this work, married had similar depression status compared to widowed or divorced and did not play a confounding role in the investigated relationship; however, it should be mentioned that no measurements of psychological stress were applied in the ACS patients. Moreover, no differences were observed between marital status groups and body mass index levels. In accord to the findings of the US Health Professionals Study, it was also observed that widowed or divorced consumed higher quantities of alcohol, compared with never-married, but increased alcohol intake was also observed among married people. 


\section{Limitations}

One limitation of the present study is that it enrolled only survivors of ACS. Thus, lifestyle or other information from patients who died during the first days of hospitalization was not available. Another limitation is that the analysis between marital status and prognosis of patients was focused only at one point in time (ie, changes in marital status were not evaluated). However, in Greece the rate of remarriage in the investigated age group is considered very low (ie, $<5 \%$ ). Moreover, no instruments of psychological stress were used, thus lifetime psychological well-being was not evaluated in relation to marital status of the patients. Finally, due to the small number of enrolled women, and the even smaller number of events, no gender-specific analysis was carried out.

\section{Conclusions}

The results from the present work suggest that ACS patients who were never-married at the time of the cardiac episode experienced higher likelihood of fatal events during hospitalization or 30-days after discharge compared with patients who were married. This finding was supported by a large cohort of patients, as well by several adjustments that were made to control for potential confounding, and emphasizes the need for special attention in this sub-group of people, especially when other age-related co-morbidities exist. However, the patterns between marital status and health are much more complex than has been previously recognized, and further work is needed in order to establish a causal pathway.

\section{Disclosure}

The authors report no conflicts of interest.

\section{Acknowledgments}

The authors would like to present and thank the field investigators of the "GREECS" study: Peter Stravopodis, Yannis Kogias, Theodoros Gialernios, Constandina Massoura, George Papanagnou, Antonis Karanasios, Labros Rizos, Michalis Mparmparoussis, George Kassimatis, Skevos Sideris, and Nick Daskalopoulos for their support in the clinical evaluation and Alexander Chalamandaris for the database management.

\section{References}

Alpert JS, Thygesen K, Antman E, et al. 2000. Myocardial infarction redefined - a consensus document of The Joint European Society of Cardiology/American College of Cardiology Committee for the redefinition of myocardial infarction. Eur Heart J, 21:502-1513.
Birkenaes AB, Sogaard AJ, Engh JA, et al. 2006. Sociodemographic characteristics and cardiovascular risk factors in patients with severe mental disorders compared with the general population. J Clin Psychiatry, 67:425-33.

Booth A, Amato P. 1991. Divorce and psychological stress. J Health Soc Behav, 32:396-407.

Eng PM, Kawachi I, Fitzmaurice G, et al. 2006. Effects of marital transitions on changes in dietary and other health behaviours in US male health professionals. J Epidem Comm Health, 59:56-62.

Focus on the Family. 2006. Focus on social issues: Marriage and family [online]. Accessed on August 3, 2006. URL: http://www.family.org/ socialissues/Marriage/.

Gallo LC, Troxel WM, Kuller LH, et al. 2003. Marital status, marital quality, and atherosclerotic burden in postmenopausal women. Psychosom Med, 65:952-62.

Hemstrom O. 1996. Is marriage dissolution linked to differences in mortality risks for men and women? J Marriage Fam, 58:366-78.

Hu Y, Goldman N. 1990. Mortality differentials by marital status: An international comparison. Demography, 27:233-250.

Idler E, Angel R. 1990. Self-rated health and mortality in the NHANES-I epidemiologic follow-up study. Am J Public Health, 80:446-52.

Mendes de Leon CF, Appels AW, Otten FW, et al. 1992. Risk of mortality and coronary heart disease by marital status in middle-aged men in The Netherlands. Int J Epidemiol, 21:460-6.

Mirowsky J, Ross C. 1986. Social patterns of distress. Ann Rev Soc, 12:23-45.

Murphy M, Glaser K, Grundy E. 1997. Marital status and long-term illness in Great Britain. J Marriage Fam, 59:156-164.

Nilsson PM, Nilsson JA, Ostergren PO, et al. 2005. Social mobility, marital status, and mortality risk in an adult life course perspective: the Malmo Preventive Project. Scand J Public Health, 33:412-23.

Pate RR, Pratt M, Blair SN, et al. 1995. Physical activity and public health. A recommendation from the Centers for Disease Control and Prevention and the American College of Sports Medicine. JAMA, 273:402-7.

Pitsavos C, Panagiotakos DB, Antonoulas A, et al. Greek study of Acute Coronary Syndromes Study Investigators. 2005. Epidemiology of acute coronary syndromes in a Mediterranean country; aims, design and baseline characteristics of the Greek study of acute coronary syndromes (GREECS). BMC Public Health, 5:23.

Radloff LS. 1977. The CES-D scale: A self-report depression scale for research in the general population. Appl Psychol Measure, 1:385-401.

Slater PE, Ever-Hadani P, Harlap S, et al. 1988. The relationship of sex and marital status to coronary heart disease risk factors in Israeli medical students. J Am Coll Health, 36:323-7.

Steptoe A, Owen N, Kunz-Ebrecht SR, et al. 2004. Loneliness and neuroendocrine, cardiovascular, and inflammatory stress responses in middleaged men and women. Psychoneuroendocrinology, 29:593-611.

Umberson D. 1992. Gender, marital status, and the social control of health behavior. Soc Sci Med, 34:907-17.

Venters M, Jacobs DR Jr, Pirie P, et al. 1986 Marital status and cardiovascular risk: the Minnesota Heart Survey and the Minnesota Heart Health Program. Prev Med, 15:591-605.

Weiss NS. 1973. Marital status and risk factors for coronary heart disease. The United States health examination survey of adults. Br J Prev Soc Med, 27:41-3.

Williams DR, Takeuchi D, Adair R. 1992. Marital Status and Psychiatric Disorders Among Blacks and Whites. J Health Soc Behav, 33:140-57. 